\title{
Improving workplace expertise to meet increasing customer requirements: the impact of training
}

\section{Jan N Streumer and Marie-José Calon}

\begin{abstract}
This article focuses upon the training of engineers at a factory producing integrated circuits. Inadequate use of statistical process techniques by the engineers meant that the production process was not being optimised in the context of increasing customer requirements. A training needs analysis was undertaken and a training programme was developed, implemented and evaluated. The results of this programme are presented and conclusions drawn.
\end{abstract}

\section{Introduction}

Performance improvement efforts need to be connected to business goals. It is the business goal of a division of an IC-factory belonging to a global electronics company, to produce integrated circuits (ICs). The management of the division concerned is continuously confronted with the increasing demands of clients: ICs that meet high quality standards at a competitive price.

The production of ICs is a cyclic process consisting of a large number of operations, the results of which are measured (where possible). The measurements are analysed by the process and equipment engineers using

$\square$ Jan Streumer is Associate Professor in the Department of Curriculum, Faculty of Educational Science and Technology, University of Twente, Netherlands. Marie-José Calon is a Project Manager at a large Dutch multi-media company, MediaVision, in the Netherlands.
DATA, a statistical analysis system. This process analysis is intended for the investigation of process problems and for monitoring and optimising the production process.

The automation section, which is responsible for the DATA system, had stated that DATA was being used by too few of the engineers and that, when used, it was not being employed optimally. The management had the impression that DATA should be more intensively used in order to meet the ever increasing client requirements.

This study sought to determine the causes of the problem, how the problem could be classified, and whether there was a need for training. On the basis of needs analysis, it was concluded that the situation involved a performance problem for which a solution had to be found. The needs analysis has also led to a clear description of the need for training among the target group. Subsequently, on the basis of these results, a training pro- 
gramme has been developed, implemented, and evaluated.

The following questions were central to the needs analysis:

1. In consideration of the organisational goals, are there sufficient reasons to accept an intensification of the use of DATA?

2. Does intensification fit with the target group's work-methods?

3. Can the problem be classified in terms of skills and attitudes?

In order to answer these questions, first, the system's possibilities were analysed. Subsequently, it was considered how DATA was used in a comparable product division within the same IC-factory; and finally, whether or not the task description for the optimal use of DATA (developed within the comparable production division) would be appropriate for the target group was considered. Experts in system-technology, process-technology, and statistics were consulted for the system analysis and for the description of the optimal use of DATA. A definitive description of the optimal use of DATA was compiled by means of analysing the tasks of the target group which have bearing upon the (future) use of DATA. On the basis of these results, the decision was made to develop and implement a training programme.

From evaluation of the training, it appears that the use of DATA has increased significantly. Based on assessment of the trainees' reactions, the training appears to have been judged positively.

The present study is set in a large Dutch factory where ICs are produced in various product divisions. An IC can be imagined as a stack of minuscule layers of different materials that form a chain of transistors. The IC is the result of many physical and chemical procedures that are performed on a wafer. Since these processes are extremely interdependent and interactive, it is necessary to control all stages of production very carefully.

In earlier years of IC process technology, little knowledge was available about the effect of certain process on its resulting product. In order to monitor the process, measurable results were sought toward facilitating revision (and improvement) of the process itself. The measurable results were found in the form of in-line and $\mathrm{pcm}^{*}$ parameters which offer after-the-fact images of not only the measured (micro)process, but also the entire process as a whole. On the basis of this information, the (sub) process and the process could then be reconsidered. The initial choice for in-line and pcm parameters was logical, since they measured the wafers themselves, thus yielding direct information over the final product.

Increased consideration of available information regarding the production characteristics also yield growth in the arena of process control. Instead of using values to correct the apparatus after-the-fact, the values of the managed parameters were later used to regulate parameter trends and (at critical moments) implement corrective measures. This manner of process control was given the term 'statistical process control' (SPC).

In order to perpetuate SPC, insight into the parameter characteristic(s) is essential. Not only is it necessary to be able to judge if a pattern or measured value is acceptable, it is also necessary to be able to determine if such a pattern or measured value requires adjustment of the apparatus. In order to help with making these judgements, so-called 'control cards' are used. The control card is a graphic in which the trend of measured values is recorded and can be read whenever a point falls outside of the pre-established control parameters. If a point falls outside of the control parameters, then the cause must be found and adjustments must then be made on the apparatus.

The above information indicates that SPC is a manner of working in which the process is guided via a visual assessment of a pattern or measured values. Adjusting the process is then realised by fine tuning (for example) equipment settings.

Increased knowledge about product characteristics not only allows a process to be driven based on SPC, but also a process which is driven based on the desired parameters which can unfavourably influence the final result. From a technical standpoint, management of the source (the apparatus) is preferred, rather than management of its

\footnotetext{
* Process control modules. After the production of an IC the physical properties of the IC are measured. The physical properties of an IC are the result of one or more combinations of online production processes. These processes can be measured by means of parameters.
} 
diversion (the result of the adjustment), because the former yields more pure information for process control. In addition, machine parameters can give information about the result of an adjustment which cannot be measured by in-line parameters. Additional investigation was conducted toward machine parameters which either offer additional forms of the already available information about the result of the adjustment, or machine parameters which finally replace the in-line measurements altogether.

In order to monitor and guide the production process of ICs, a statistical analysis system (DATA) was used. DATA is a component of a larger error recognition information system, and was used as a tool to support SPC.

After all processes have taken place, the final physical properties of the IC are measured (ie. the resistance of a track). At a later stage, the IC is measured to determine if it meets the requirements set out in the design specifications. The parameters which are measured in this way form the basis for process control and process optimisation. Through the use of the DATA system, statistical analyses can be performed on the data collected about the measured parameters. With the aid of this instrument, it is possible to select a batch of ICs based on certain criteria, from which the measured values of selected parameters would then be calculated. The user may indicate, for example, which statistical analysis should be conducted on which measured value (ie. variance and regression analysis). The results are then printed on control cards, upon which measured value patterns become visible. The measured value must remain within critical parameters. On the basis of information (such as client specifications) this procedure should be kept within (increasingly narrower) critical parameters.

Analyses with DATA may be conducted with a problem-oriented approach in order to determine possible causes when ICs fail to meet the necessary specifications. The analyses can also be performed for preventative purposes, in order to control the production process.

The production process of ICs consists of four clusters of procedures: 'oxidation', 'photolithography', 'etching', and 'ionimplantation and metallisation'. Two equipment engineers and one process engineer are assigned to each cluster of procedures. They are responsible for the machinery within the cluster and that part of the process with which the cluster is concerned. Process engineers who are responsible for the entire process are active within all of the clusters. Because the overall process is seen as a vertical process, the process engineers within the clusters are called horizontal process engineers and the others are called vertical process engineers. There is also a third category of process engineers: yield engineers. They are responsible for the final yield: the wafer.

\section{Problem description}

Automated Information System operators suspected that engineers did not make optimal use of the DATA system, and that this was due to the engineers' lack of familiarity with the programme.

At the management level, the impression had developed that DATA should be used more intensively, in order to meet the growing quality requirements of the clients, who demand an ever increasing degree of process mastery. It was seen as desirable that the different engineers should work more closely with one another when conducting the process analysis. The target group desired a situation in which analyses could be performed without the involvement of the Automation section. Until recently, the analysis was actually conducted by the system controllers, who did this on the basis of analysis requests from the engineers.

In addition to the engineers' unfamiliarity with the potential of the DATA analysis system, operators lacked awareness of the process technology. This, together with insufficient documentation to support its use and high staff turnover levels meant that few, if any of the remaining process and equipment engineering staff fully understood the technical complexities of the DATA system. The Automation section therefore suggested training in use of the DATA system for the equipment and process engineers to encourage their independent use of the analysis system.

The problem description which serves as a starting point for this study has been formulated as follows:

How can a situation be reached in which the process and equipment engineers can, without the intervention of the Automated Information System operators section, make more intensive use of the 
DATA system for the performance of process analysis?

\section{Needs analysis}

A description of the problem was arrived at following consultation with all parties involved (process engineers, automation system operators and management). At the management level, there was a belief that firstly, more use should be made of the DATA analysis facility, and secondly, that engineers should work more closely together on the analysis, rather than in isolation. Engineers apparently wanted to be able to carry out the task of analysis without the involvement of the automation system engineers. Previously, analyses had been requested by engineers and carried out by the Automation Section Controllers (ASCs), because the engineers did not have the necessary knowledge of DATA. However, since the ASCs did not have the relevant engineering knowledge to understand precisely what information was required, very often the required or most appropriate data was not obtained from this arrangement. As a continuation of this consultation, in which many of the opinions and suppositions of the concerned parties were expressed, it was examined whether there was actually sufficient reason to intensify the use of the DATA system (eg., is DATA the most appropriate means to achieve the chosen goal?), how an intensified approach could be characterised, whether on these grounds a training-need can be inferred, and if so, what is it? This was done because, within the product division, there was too little knowledge of the system and therefore, great value was attached to the most objective picture possible of the existing and the desired situations with respect to the use of DATA, in order to make a responsible training investment.

In order to conduct the needs analysis in a systematic manner, the levels of needs analysis of Schramade, the Organisation Elements Model (OEM-model) of Kaufman, and the objects and techniques of needs assessment of Rossett, were used [1]. In the above-mentioned methods for the conducting of needs analysis, the problem is approached from differing viewpoints. This is because they are used here in an interrelated manner. A similar approach has been used before by Ori and Streumer [2]. An important criterion in the selection of the first two methods was that a direct relation with longer term organisational goals was present in both. For the latter, the accent placed upon techniques of information collection was of importance.

On the basis of earlier research from Kirkpatrick, Schramade distinguishes various levels of needs assessment which are based on the organisational functioning and that of individuals and groups within an organisation [3]. Because symptoms of a problem often occur on different levels of an organisation, Schramade advocates conducting needs analysis which is specially intended for such situations. The problem must first be localised on one of the levels and subsequently approached from the various levels of an organisation in order to determine where the possible sources of the problem lie. The levels which are distinguished in his model are:

1. the external functioning of the organisation or organisational component;

2. the internal functioning of the organisation or organisational component;

3. the working behaviour of the personnel;

4. the knowledge, insight, attitude, and skills of the personnel.

Kaufman employs the OEM-model for conducting needs assessment and needs analysis. According to Kaufman and Valentine

\begin{abstract}
Needs assessment identifies gaps in results, places them in priority order, and selects the most important for closure or reduction. Needs analysis identifies the causes of the gaps in results so that appropriate methods, means, tactics, tools and approaches may be rationally selected for meeting the needs [4].
\end{abstract}

By using the OEM-model an exact description of the existing and the desired situation can be given in terms of inputs, processes, products, outputs, and outcomes. In this manner, the problem is approached systematically in order to include in the needs assessment all of the possible influences on the relations between the above-mentioned factors. Kaufman advocates conducting a needs assessment, the description of the existing and the desired situations in terms of the specified factors, and a needs analysis: an examination of the extent to which there is a good match between the specified factors. Rossett describes needs analysis as a:

Systematic study of a problem or innovation, incorporating data and opinions from varied 
sources in order to make effective decisions or recommendations about what should happen next [5].

She distinguishes the following research objects: optimal performance or knowledge, actual performance or knowledge, feelings of trainees or significant others, causes of the problem from many perspectives, and solutions to the problem from many perspectives. She distinguishes four techniques which must be used in order to conduct a needs assessment of the specified objects of research: extent data analysis, needs assessment, and subject matter analysis. Extent data analysis (EDA) is intended for the collection of information concerning the quantitative and qualitative results of actual job performance. Needs assessment (NA) is intended for the categorisation of opinions from various sources about the above-mentioned research objects. Subject matter analysis (SMA) is intended for the analysis, in consultation with the appropriate experts, of which knowledge and skills are required in order to function optimally.

On the basis of the above-mentioned models and methods for needs analysis, the following research questions have been formulated. In terms of the levels of Schramade, the problem occurs on the level of the workbehaviour of the employees [6]. The engineers should use the DATA system, more intensively. This seems to be hindered by a lack of knowledge of the analysis system's capabilities and applications. In Schramade's model, this is a problem source that belongs to the level of the knowledge, skill and attitude of the employees. The goal of further optimising the process mastery, can be located on level of the internal functioning of the organisation. On the basis of this classification, there are several research questions that must be answered:

Level 'internal functioning of organisation':

1. In consideration of the organisational goals, is there sufficient reason to intensify the use of the DATA system?

Level 'work behaviour of employees':

2. What are the characteristics of the existing and the desired job performance in relation to the use of the DATA system?

Level 'knowledge, skill and attitude of employees':

3. What are the employees' existing and desired levels of knowledge and skills in statistics?

4. What are the employees' existing and desired levels of skill with and knowledge of the system's capabilities and applications?

5. What are the employees' existing and desired attitudes toward the use of the system and the desired changes?

Level 'supplement to the Schramade model':

6. Is there, on the basis of these questions, indication of a training problem and a need for training?

7. How can these be characterised?

The preceding questions can be used for the initial approach to the problem. Kaufman's OEM-model is useful for further development of the questions. This model offers the possibility of examining the limiting conditions and of further specifying which factors influence the translation of organisational goals into work-behaviour. This transition seems rather large in the model of Schramade (1985 [7]). In Figure 1, the problem is approached, with the aid of Kaufman's model, from a 'process' viewpoint. For the aspects which have still not been adequately developed, the desired additional information is indicated in the form of a question. It is clear that the longer term organisational goals can only be achieved through a multiplicity of improvements, the analysis of measured data is just one of these.

A further supplement to the model of Kaufman is provided by Rossett, who concentrates on the views of the concerned parties with respect to the suspected problem and the desired changes [8]. She also pays a great deal of attention to the causes which lie at the root of the problem. Rossett gives a clear description of techniques which can be employed in needs analysis (data analysis, system analysis, task analysis and target group analysis). All these techniques have been coupled to the research questions that were formulated using the Kaufman model. Further, it has been determined for each of the techniques, which procedures will be used to collect information, and which of the concerned parties should be consulted for this purpose.

Data analysis is applied in order to determine which parameters are analysed, to what degree process analysis is conducted by the different engineers, and which of the system's possibilities are being made use of. These data are registered by the system and give an objective picture of the degree to which the system is used within the present work-situation.

In order to determine the system's capabilities, purposes, and applications, a system analysis was conducted. The goal of the system analysis was to describe the system 
When, why and to what degree is DATA used for analysis?

How is DATA used for analysis?

Which problems or difficulties occur?

How are the data from DATA interpreted?

Can DATA be and is it being used for the analysis of control charts?
1. Which parameters are measured and how are they measured?

2. What are the possibilities of

DATA?

3. The target group has no access to DATA. What are the characteristics of the target group?

4. Which statistical knowledge is necessary and which is present?

5. Which system knowledge is necessary?

6. How can the attitude be characterised, and what are its causes? How can these causes be removed?

7. The parameters do not all have a control chart

\section{PROCESSES}

8. How are the parameters analysed?

9. How are the control charts analysed?

10. Which task distribution presently exists?
1. Useful and trustworthy data in DATA

2. Useful and trustworthy analysisresults

3. Access to DATA for process and equipment engineers. Under which conditions can this be realised?

4. Adequate level of statistical knowledge

How should the data of DATA be interpreted?

5. Adequate level of knowledge concerning the system possibilities

6. Positive attitude with respect to DATA

7. All parameters have a control chart for the running of SPC

How should DATA be used? purposes should DATA be used?

How should DATA be used for the analysis of control charts during SPC?

8. Which analyses must be performed?

9. Analysis of the control charts during the SPC

10. Cooperation between process and equipment during analysis tasks

\section{PRODUCTS}

11. Not all the parameters meet the process mastery

11. A contribution to the optimisation of process mastery

What contribution can be delivered through analysis? criterion

\section{OUTPUTS}

12.

13.
12. A contribution to a qualitatively more valuable product

\section{OUTCOMES}

13. A contribution to a reduction of client complaints

Figure 1: Problem analysis 
knowledge and skills which the users need in order to make optimal use of the DATA system. In consultation with a system expert (from a different product division), the system's capabilities were illustrated in a graphic lay-out, and, using this information, it was determined for which purposes the system's capabilities could be used and in which situations they could be applied. On the basis of the system's capabilities and the system output it was subsequently determined which statistical knowledge and skills the users should possess; and a schematic illustration of potential outputs or elements was created. Per element, it was illustrated which statistical quantity of the output should be analysed. On the basis of the produced diagrams, it was determined into which coordinated statistical elements the data to be interpreted from the diagrams should be divided. During the designing of the training, elements were used for the formulation of the goals.

Within the product division where the system expert is employed, the DATA system appeared to be used intensively and without problems. Therefore, a task analysis was conducted in that product division in order to determine how the DATA system could be used for tasks which are connected to analysis activities. As an expert in this area, the head of process-engineering was consulted, and it was analysed as to which tasks and (for each task) in what manner DATA should be used. Within the product division for which the training was being developed, the desired degree of expertise and knowledge was not present. Because the situations in the two product divisions were not totally comparable, an analysis of tasks for which the DATA system was or could be used was also conducted with the target group (the process and equipment engineers). It was determined which goals the DATA system was currently being used for (or would be used for in the future) by the target group, how DATA was being used, and to what degree the task description of the comparable product division was applicable to the analysis tasks of the target group. From the viewpoint of acceptability, the task analysis also offered the possibility at an early stage of involving the users in the desired changes. On the basis of both task analyses, a desired task description was compiled for the different groups of engineers in the target group for the analysis tasks in which the DATA system can be used.
As a component of Rossett's needs analysis, a target group analysis was performed. The most important characteristics of the analysis were the following: work experience, previous training, having attended statistics courses, knowledge and skills concerning the system's possibilities. On the basis of his work experience with the users, the statistician made an estimate of their level of statistical knowledge. Using the system output, it was also determined how the system output data is interpreted, and whether further explanation of the statistical quantities and their interpretation would be desirable.

The results of the needs analysis can be summarised as follows:

1. Although the problem was originally caused by inadequate facilities (the users had no access to the DATA system) and it was increasingly manifested as a communication problem between the system controllers and the users, the problem can be characterised as a training problem. In other words, there are, in the target group, deficient knowledge, skill, and attitude aspects in relation to the use of the DATA system.

2. The DATA system is used primarily by the vertical engineers, while it offers useful possibilities for the entire target group. The task description, which was compiled in response to the needs analysis, indicates all functions of the target group and at which moment and for which purpose the DATA system can be used. Because the use of the DATA functions as a support to the analysis tasks, it can be concluded that an intensified usage fits in the job description.

3. In order to implement an intensified application of the DATA system, the relation between their own tasks and the use of DATA and the interpretation of the data must be made clear to all engineers. Although one of the function groups within the target group possesses better knowledge of the system's possibilities and applications than the other groups, differentiation does not seem desirable, because more cooperation from within the different function areas is desirable for the performance of process analysis.

4. The DATA system may be used to support a process-execution based upon statistical process control, both by increasing 
the insight into the characteristics of the parameter, and by conducting problem analyses in response to a pattern of measurements on the control chart. Also, from statistical process control considerations and therefore indirectly organisational goals, there is enough reason to implement an intensified application of the DATA system.

To summarise, a training programme in the use of DATA has the following goals:

a. to obtain knowledge concerning the system's possibilities, purposes, and applications in respect to the tasks of the target group, in order to use the possibilities correctly and in a goal-oriented manner;

b. to obtain knowledge of the statistical quantities, terms, concepts, symbols, and data that are reported in the system output, in order to interpret the data correctly;

c. to attain skillfulness in the application of the system's possibilities and the interpretation of the produced data;

d. to attain skillfulness in entering analysis request into the system;

e. to develop an attitude in which the readiness to use DATA for the execution of tasks is apparent.

The results of the needs analysis and the above-mentioned training goals were presented to the management, after which interest in a training programme in the use of DATA was expressed, and the decision was made to design the training programme.

\section{The training programme}

For the development of the general training goals into a design for a training programme, the methodology that Posner and Rudnitsky prescribe for the development of curricula was applied [9]. It is clear that knowledge of the system output, the statistical quantities which appear in the output, and the system's possibilities are necessary conditions for the interpretation of the produced data. These knowledge and skill aspects are essential if the users are to apply DATA. In terms of the cluster principle of Posner and Rudnitsky, the relation is based upon the learningrelated principle. In other words, the relation between the objectives is based upon the manner in which the learning process occurs. On the basis of the cluster principle, the objectives can roughly be divided into two categories:

1. knowledge of the system's capabilities, purposes, applications, output, and statistical quantities, and skillfulness in the interpretation of data;

2. skillfulness in the practical application of the system's capabilities and in entering analysis requests.

The distinction is based upon the assumption that the knowledge and skill aspects which are named in the first category form necessary preconditions for the skill aspects of the second category.

The DATA training programme consisted, in the order presented, of the following:

- A preliminary statistics course for the reactivation of prerequisite statistical knowledge. What this basically implies is that the preliminary course is constructed around statistical topics that are considered to be prerequisite knowledge. The topics are presented in a logical (or a content-determined) sequence. As well as a theoretical explanation of the concepts, their practical use is demonstrated using examples from the production process. Duration: two hours a week for three weeks.

- The DATA core training programme in which, using the system output, knowledge is obtained concerning the system's possibilities and applications, and skill is developed in the interpretation of the system's possibilities. The system output is a central component in order to train the users in an application-oriented manner.

The DATA core training programme was filled in with a selection of examples of system output which were oriented towards the specific tasks of the different function-groups. In this way, the design differentiated between the viewpoints from which the different function groups conducted their analyses. Because the groups must cooperate with one another in order to conduct the analyses and because an analysis cannot be approached from just one viewpoint, differentiation was only desirable to a limited degree. Within the sequencing of the task-oriented system output, the selection possibilities and the statistical interpretation were treated. For the sys- 
tem possibilities, the decision was made to zoom in from the total picture toward the specifics, and, for the statistical topics, to use content-dependent sequencing. Duration: two hours a week for six weeks.

- A practical application section in which DATA, as a support for SPC and as a tool for practical use, is practised and brought into relation with the practical work situation.

The application section was filled in after a training in entering analysis requests, by giving task-oriented assignments which had the purpose of:

1. analysing the conditions under which SPC operates;

2. analysing the control chart of a parameter;

3. analysing the interrelationship between parameters.

Because it concerned real practical assignments the learning time could be reduced to a minimum, and the analysis results had a high practical value. Duration: two hours a week for three weeks.

- A system manual, which was intended as a guide for the performance of process analysis.

A full gathering (20 people) was decided upon for the organisation of the preliminary course in statistics and the complete course. For the application section, work-groups of four people were formed, in which all of the engineering functions were represented. The implementation of the training took in total two hours for 12 weeks.

\section{Evaluation framework}

The evaluation of the training had, on the one hand, a formative goal (the revision of the course components), and on the other hand, a summative goal (the assessment of the transfer-effect of the training). In order to meet these goals, an evaluation in the form of a measurement of reaction was performed.

Although, from an educational point of view, testing is a generally useful and trustworthy method of determining the learning effect, it was not used here. Within the electronics company concerned, it is not customary to use a test as a gauge of learning effect from this sort of training (which is oriented towards job performance), because it can have a threatening effect. Also, it ignores the possibility that users might 'settle into' the system with time. The reaction measurement which was used as an alternative, took the form of a questionnaire in which propositions were given to the participants in the course and the head of process engineering. Judgements were requested in the form of a five point scale. The objects of evaluation which were integrated into the propositions, were concerned with:

1. the prerequisite knowledge;

2. the course objectives;

3 . the course content;

4. the course materials;

5. the presentation and work-form.

Along with the reaction measurement as a more objective method, an evaluation of the frequency of the use of the DATA system took place (before, during and after training). The goal of this evaluation was to form a judgement concerning the transfer-effect of the training. The evaluation of the frequency of use relied on hard data: the system records at what moment and by which person an analysis request is made. The analysis requests from which the frequency of use is measured can be identified as non-periodic analysis requests, which are, for example, conducted as an interim check, as a response to a signalled problem in the control chart, or as a response to a partial or complete break down (these are also referred to as 'problemoriented analyses'). Periodic analyses are checks which are performed every two weeks, every month, or every three months on a fixed number of parameters, independent of what occurs with the process. For the most part, these periodic analyses were previously conducted by vertical process engineers. During the evaluation, the frequency with which the problem-oriented analyses were conducted was measured, and it was determined whether periodic analyses were conducted by the various function groups within the target group. In contrast to the non-periodic analyses, the frequency of the periodic checks was not determined, because these checks were done for a constant number of parameters.

\section{Evaluation results}

\section{a. Reaction-measurement}

From the evaluation results of the DATA training programme and the applications, it appears that the training, and especially the 
application section, was generally judged positively. Because the training participants demonstrated a critical attitude during the training, the written reactions are assumed to have a considerable degree of validity. The most important results are (Likert scale with 5 points: 1 is lowest score; 5 is highest score): good match with previous (statistical) knowledge and skills (item 1; 4.5); knowledge acquisition (item 2a-2h; mean 3.8); useful to follow training programme (item 4.5); relation to every day work-life (item $5 ; 4.5$ ); content of course in relation to achievement level of trainees (item 8a; 4.3).

The response to question $1 \mathrm{~b}$ reflects the heterogeneous composition of the target group. With regard to questions 2 a through $2 \mathrm{~h}$, in which the training objectives are handled, it should be noted that the dispersed contribution of the answers was mainly caused by the variation in the frequency of use (question 1b) before the course began. Also, it seems that the responses which listed answer category 0 were consistently given by the same person. When these results are omitted, then the responses of the participants show an (ever closer) resemblance to those of the head of processing. See Table 1 for more detailed information.

\section{b. Transfer-effect}

For the non-periodic analyses, the frequency of use is determined by recording the number of processed analysis requests per person and per period. The data are subsequently displayed per engineer function.

For the interpretation of the data, it is of interest to know that the frequency of use is not comparable between function groups, because one function may confront more situations which call for the use of the DATA system than another function group. In total six frequency measures have been carried out (period 1 to 6), respectively in April, May, June, August, September and November (see Figure 2). It should be noted that the training programme began on 1 May. The application part of the course started early June and ended early July. During the second half of June and the first half of July, the frequency of use was strongly influenced by the application part of the training course. After this period, from the second half of July until the beginning of September, a staggered vacation period took place. Although the use of the DATA system was higher in this period than in the period before, it does not provide a realistic picture of the use of DATA, because of the vacation-effect. A more accurate idea of the transfer-effect can be obtained through comparison of the data from periods 1 and 2 (before the beginning of the DATA training programme and the application section) with the data from periods 5 and 6 which were, respectively, 2 and 4 months after the end of the training.

From the data, it appears that the average use, measured for all functions, has increased. It appears that the previously recommended caution should also be applied here, due to the fact that strong fluctuations in use, particularly among the horizontal process engineers, can be perceived. One possible explanation for the drop in the frequency for the concerned function group in period 5, could be that the daily work, which has a direct influence upon the continuity of the process, was very demanding for this particular function group during this period, and, therefore, the analysis tasks were pushed to the side. This explanation seems acceptable, because the drop was recorded after a vacation period (after which lost time has to be recovered through more intensive work) and because this particular function group works close to the production process.

With respect to the periodic analyses, it appears that these analyses are performed by all of the engineers after the total training programme.

\section{Discussion}

This study shows that a systematic needs analysis procedure by which the problem is assessed leads to a sound problem definition. Such a needs analysis procedure should lead to the description of the necessary knowledge, attitudes and skills of the employees, a description of the improvement criteria of the work behaviour of employees for which the knowledge, skills and attitudes are prerequisites. And of course criteria which can be applied to describe the direction in which the organisation should move to achieve the goals set. The improvement of the work behaviour of employees, eg. their performance on the shop floor, is the key to the success of the organisation.

On the basis of such a solid problem definition the design, implementation and evaluation of the DATA training programme has been carried out. The DATA training programme is a tool for the target group to use 
Response participants $(\mathrm{n}=15)$

Item context

mean standard deviation

1a. Good match with previous statistical knowledge

1b. Usage of DATA before training programme (never $\rightarrow$ frequently)

2a. Knowledge acquired of:

*Selection possibilities

2b. *Kind of system output

2d. *Terms and statistical quantities

2e. *Skills attained in interpretation

2f. $\quad$ In performing supplementary computations

2g. * In entering analysis request

2h. $\quad{ }^{*}$ More applications

3a. New information in training programme

3b. Familiarity of information

(not familiar $\rightarrow$ very familiar)

4. Useful to follow training programme

5. Relation to every day practice

6. Relation training programme content vs. examples

7. Insightful examples

$4.1 \quad 0.7$

8. Treatment of subject matter matched with knowledge level

9a. Pace easy to follow

9b. How was pace?

in setting up problem analyses. In addition, it is an aid for the support of statistical process control and statistical manufacturing control. The use of the DATA system is, in and of itself, not a goal, but a means with which to reach other goals [10]. The training programme aimed at the usage of the DATA system has been judged positively by the trainees (engineers) and has contributed to an intensified usage of problem oriented and periodic DATA analysis for especially vertical, yield and equipment engineers. The contribution of horizontal process engineers consisted mainly of a more intensive periodic application of the system. The application of
DATA is not a objective in itself, but a means to achieve higher order objectives, namely the application of DATA as a tool for the support of Statistical Process Control (SPC).

Following training in the use of the DATA system, the initial part of the full training scheme is completed. From the evaluation it seems that the assumption may be made that knowledge and skills necessary to use the DATA system are present. It may also be assumed that, with better insight into the potentialities, goals of use and applications for this system, the engineers are therefore more prepared to put it to use in their everyday situation. These factors, however, do not 

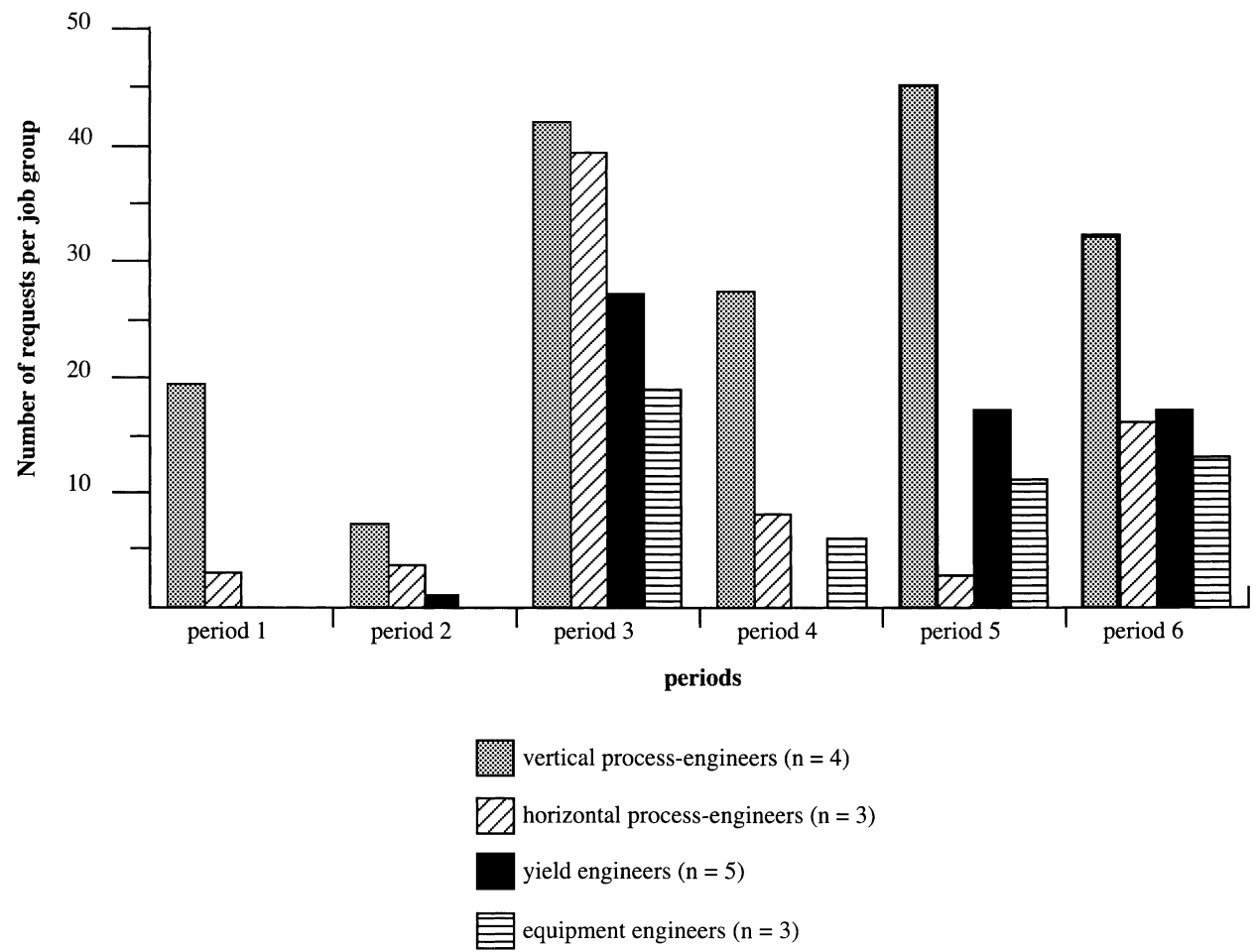

Figure 2: Non-periodic analyses requests per job group

guarantee a further increase in the use of DATA; follow-up on the training is crucial to meeting this aim.

In order to use the DATA system, some of the engineers must work and think differently from the ways to which they are accustomed. With regard to transfer and retention of the new knowledge and skills, it appears that in addition to individual initiative, influence of the engineer's environment is necessary. Periodically asking the head of process engineering for the statistical analysis results might be a way to guide this. Also, toward preventing problems with the use of the DATA system, such a technique might be applicable. A similar stimulation appears to be necessary to make the use of the DATA system an assumed part of the daily routine. This notion is confirmed by, among others, Noel and Dennehy who suggest that the transfer of learning to the workplace will not be achieved without the stimulation and interest of the supervisor [11].

To advance transfer, on-the-job training may be effective. For problems or issues that may be encountered by more than one individual, it may be useful to offer additional training in the use of the DATA system guidebook, so that individuals may learn how to solve their own problems. By discussing the problems, together with colleagues, the use of the DATA system and the interpretation of information would then be incorporated into the behaviour of engineers in an effective manner. On the basis of knowledge and skills acquired through this type of training, subsequently practical assignments may be given to support a user in reaching a higher level of mastering and application of the DATA system.

When using the system guidebook, a few caveats should be mentioned. It cannot be assumed that such a guidebook will be completely self-instructional. It is often said that a shortcoming of such guidebooks is that they are incapable of offering remediation or feedback to the learner about her/his activities. In addition, it is also often suggested that new learners do not work with these guidebooks alone, they are suggested for use as reference material only (and in this area, they are very helpful). A combination of guided instruction with self-instructional materials appears to be the most preferable situation. 


\section{References}

1. Schramade, P., 'Strategieën voor behoeftenbepaling' (Strategies for needs assessment) in Wetenswaardigheden, B.5.2.1., maart. 's-Gravenhage: ROI/CIVoB, 1985; Kaufman, R. and Valentine, G., 'Relating needs assessment and needs analysis', Performance and Instruction, Nov./Dec. 1989; Rossett, A., Training Needs Assessment, Englewood Cliffs, Educational Publications, 1987.

2. Ori, H. R. and Streumer, J. N., 'Van behoeftenbepaling tot opleidingsplan: het ontweerpen van een post-MTO-opleiding Halfgeleidertechnologie (From training needs assessment towards a training programme for IC- technology), Opleiding and Ontwikkeling, 2:6, 1989.

3. Kirkpatrick, D. L. (ed), Evaluating Training Programs, Alexandria: ASTD, 1975.

4. Kaufman and Valentine, op. cit., p. 11.

5. Rossett, op. cit., p. 3.

6. Schramade, op. cit.

7. Ibid.

8. Rossett, op. cit.

9. Posner, G. J. and Rudnitsky, A. N., Course Design, New York/London, Longman, 1986.

10. Schramade, op. cit. level 2.

11. Joel, J. H. and Dennehy, R. F., 'The learners manager: the bridge from classrom to workpla-

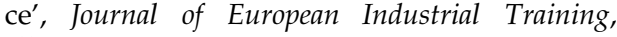
15:6, 1991 